\title{
OPEN-SCIENCE SPACE ISSUE: \\ CALIBRATION OF MEASURING CHANNELS OF NON-DISMANTLING CYBER-PHYSICAL SYSTEMS
}

\author{
Dr. Sc., Prof. Bohdan Stadnyk; Dr. Sc., Prof. Vasyl Yatsuk, Dr. Sc., \\ Prof. Mykola Mykyichuk, Dr. Sc., Prof. Svyatoslav Yatsyshyn, Lviv Polytechnic National University, Ukraine, \\ E-mail: slav.yt@gmail.com \\ Dr. Sc., Prof. Tomas Fröhlich, Ph.D., Lead. Res. Rostyslav Mastylo, \\ Technical University, Ilmenau, Germany
} \begin{abstract}
System, Calibration, Metrology 4.0
\section{Introduction}

The multifaceted development of modern society is manifested not so much in the formation of new technologies, as in their maximum environmental friendliness and the possibility of assimilation by society. This is expressed in the approximation of the conditions of study and work to home and intensified in a prolonged pandemic. Therefore, the EU focuses in its efforts, expressed in the Horizon Framework Program (20212025 (7)), on science-intensive IT projects, including the European segment of cloud technologies [1]. In this case, one of the main foundation pillars is metrology, as a scientific and applied means of achieving and implementing the reliability of the design and operation of cyber-physical systems (further - CPS) [2].
\end{abstract}

Abstract. The analysis of the concept of Open-Science Space is carried out. The existence of ways to achieve reproducibility and traceability of research results performed by a group of worldwide situated Cyber-physical system operators/supervisors is shown. Ways to ensure the efficient operation of Cyber-physical systems as complex technological nondemountable objects with high requirements for metrological characteristics have been studied. To develop the scattered cyberphysical systems, the portable stable-in-time code-controlled measures of physical quantities have been studied. They have to be metrologically confirmed in the laboratory before the delivery to the site of the measuring subsystem for its calibration.

Key words: Open-Science Space, Cyber-Physical System, Metrological characteristics, Non- Dismantling Cyber-Physical

\section{Drawbacks}

This approach is due to the narrow focus of the above development and is designed to expand the scope and implementation of a row of projects in technology. Not all its areas are equipped with modern measuring instruments, since from the moment the problem is identified to the appearance of the measuring instrument, years may still pass.

\section{The Aim of the Article}

The work aims to analyze the ways of formation of Open-Science Space and achieve reproducible and traceable results of high-precision research based on the development of small highly stable, metrologically validated code-controlled measures of physical quantities delivered to the operation place of the scattered cyberphysical system for its non-demountable calibration.

\section{Forms of development of Open-Science Space in information technologies}

\subsection{The AMASS and Other Projects}

There is a path of Open-Science Space proposed by the European Commission, the creation of which can provide the research by scattered groups of scientists. For example, the University of Arizona is proposed a research collaboration platform available to researchers. all stages of research [3]. This path contributes to:

- better management of the results obtained, including those obtained on the same type of installations mounted at remote sites of different CPSs;

- further development and implementation of Metrology 4.0, resulting in the measurement and control results reliability of complex technological processes;

- creation of conditions for the development of unique measuring instruments and their rapid implementation by a spontaneously created scientific and technical group.

An example is the AMASS open-source ecosystem project. This ecosystem includes an open-source tool platform that supports the main CPS assurance and certification activities, external means with added-value features, and an open community of developers and users. The platform integrates existing solutions for system modeling, process engineering, and compliance, and argumentation management [4]. The interest in open source means for assurance and certification is growing and different initiatives have been launched. The project supports and fosters the development of the spectra of open-source and openly developed the dispersed CPS selectively required functionality to the scientificIt allows for the management and sharing of projects at based on the Metrology 4.0 [5] that provide the 
educational community. This product can serve as the foundation pillar for a certain project of the particular user. The latter acquires the ability to solve his specific problem of the unique issues with the help of the mentioned project. It needs only to participate in the considered issue by linking and describing the user's purpose of his scientific interest that consists in measuring or/and controlling the specific characteristics which cannot be fixed, described, and reproduced by the wellknown techniques.

We also conduct the user's studies that include first the measurements, control, and management [6], then obtaining the measurement data and their processing, and finally receiving and analyzing the desired property with acceptable metrologically reliable indicators and even risk. The fulfilled together visualization makes it simpler to integrate measurement hardware from any vendor, verify the measurement software, develop data analysis algorithms as well as design local or/and dispersed CPSs.

\subsection{Project for Development of Biomedical}

\section{Studies}

It was offered by [7] the certain way of developing and implementing scientific and technological achievements based on Metrology 4.0. A universal tool has been created, replicated in small-scale production, and transmitted to the scientific community, even with software for scientific biomedical research. This approach forms a good basis for the creation of Open-Science Space. Let's consider it in more detail before proceeding to our research. Like some similar products the considered open-source hardware-software product is based on the single-board microcontrollers and microcontroller kits for the design of digital measuring instruments and actuators as the obligatory components of the CPSs.

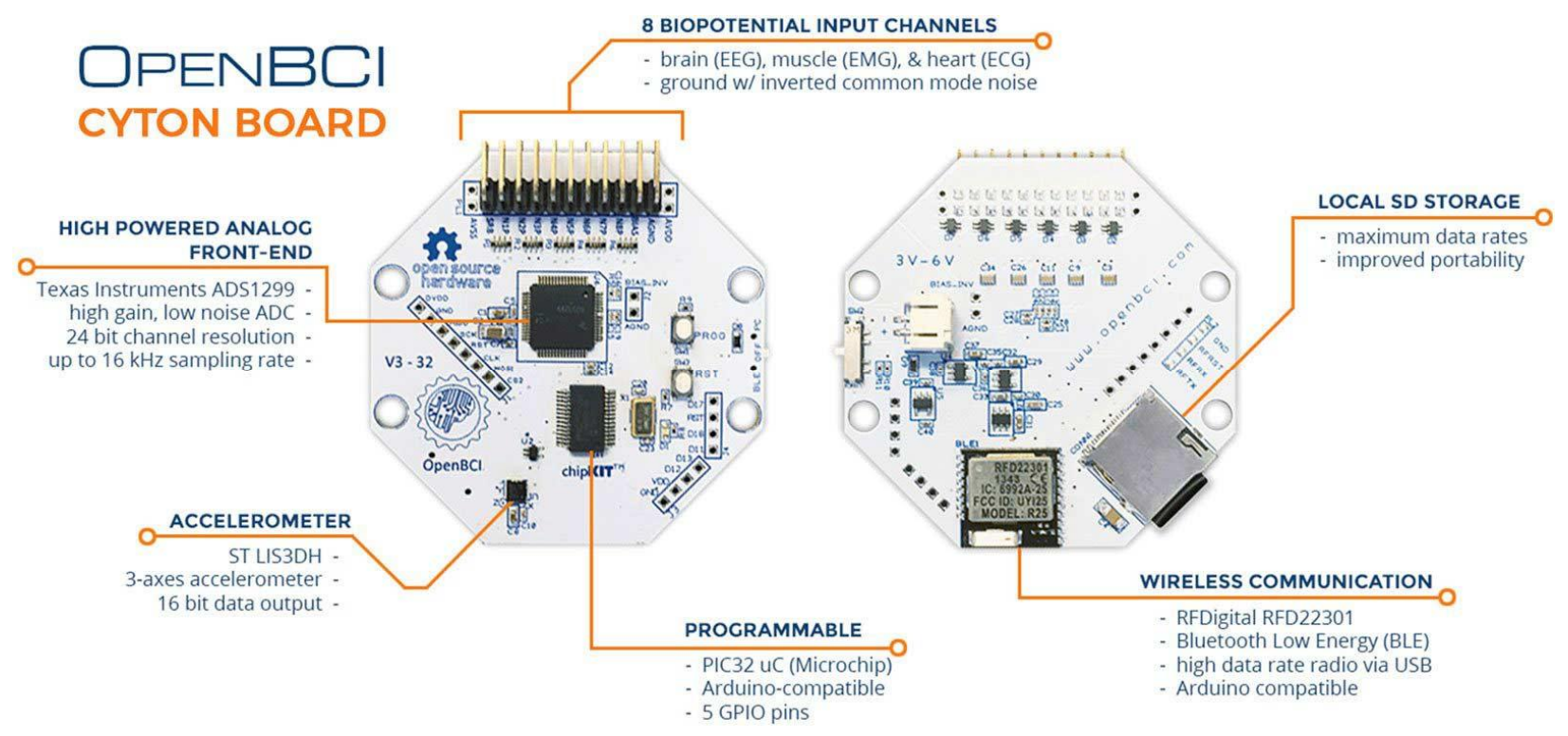

Fig. 1. The OpenBCI Cyton Board [7]

OpenBCI Cyton Board is an Arduino-compatible, 8-channel neural interface with a 32-bit processor. At its core, the mentioned board implements the PIC32MX250F128B microcontroller, giving it lots of local memory and fast processing speeds. The board comes pre-flashed with the chipKIT ${ }^{\mathrm{TM}}$ bootloader, and the latest OpenBCI firmware. Data is sampled at $250 \mathrm{~Hz}$ on each of the eight channels.

The OpenBCI Cyton Board can be used to sample brain activity (EEG), muscle activity (EMG), and heart activity (ECG). The board communicates wirelessly to a computer via the OpenBCI USB dongle using RFDuino radio modules. It can also communicate wirelessly to any mobile device or tablet compatible with Bluetooth Low Energy (BLE). The board and dongle come preloaded with the latest firmware, making OpenBCI accessible to researchers and developers with little to no hardware experience.

Due to the leak of metrological insurance and a few other reasons, the board/kit is not considered as a medical device nor is it intended for medical diagnosis; it is intended for use for engineering, development, demonstration, or evaluation purposes only.

\subsection{Development of virtual metrological} instruments as the OSS basis

The Department of Information-Measuring Technologies of Lviv Polytechnic National University has launched a similar project, based on LabVIEW - a platform and development environment for the visual programming language [8]. On this basis, there were developed the following virtual metrological tools: 
- Mean of control and regulation of temperature and time regimes of materials processing (Prof. I. Mykytyn);

- Mean of high-precision weighing and dosing (As-Prof. R. Ivakh);

- Mean of precision measuring the electric signals (Prof. O. Kochan);

- Mean of control of feeding vegetables, and also their pollution by the impurity of heavy metals (Ph.D. student I. Midyk);

- Mean of monitoring, prevention, and treatment of age-related and traumatic changes in the human limbs circulatory system (Prof. S. Yatsyshyn).

All of them are virtual metrological instruments consisting of control objects equipped with smart sensors; with hardware in the form of an I/O unit and the appropriate software installed in the PC linked to a particular end-user via the Industrial Internet. The latter does not need to manufacture the considered mean, but only connect the controlled object and provide its power. In such a way several users build OSS. However, data processing requires the implementation of the following steps regarding their metrological reliability.

\section{Metrological traceability and reliability of information-measuring subsystems}

We offer below a similar, metrologically-based way to provide worldwide scientists with access to OSS. It is based on the fact that all the above methods and means of forming OSS form mainly a qualitative vision and transfer of parameters of controlled processes. The obtained results are difficult, if not impossible, to consolidate and generalize, as they relate to different settings, taken by different experimenters and often at slightly different objects. Even the same biological object - a human - changes in several hours and his study performed on the same equipment can give significantly dissimilar results.

The intermediate conclusion was known before: the means used for measurement and control must be metrologically reliable. This should be provided by the relevant service within one country or several countries. Even with the normal operation of metrological services for complex scientific and technical facilities, which include CPS, the latter is almost impossible to check metrologically. To fulfill this, it needs to stop the process for a long time. At the same time, the dismantling and delivery of such equipment for verification of metrological characteristics do not guarantee their improvement when re-installing the tested means in the CPS since the transportation and installation worsen these characteristics by almost an order of magnitude. So, to ensure enhanced metrological reliability since 2000 there were noted the development of the so-called built-in measures of the particular characteristics. One of such first measures was the measure of electrical resistance [9]. In the United States alone in 2000, their number was about 100.

\subsection{Traceability of measurements}

Requirements for measurement accuracy may differ significantly for each specific measurement task, but they must all be implemented with ensuring traceability. The measurements of the latter of the same physical quantities are the main requirement for measurement channels to achieve data comparability, regardless of what methods and measuring instruments and under what conditions the measurements are performed [10-11]. In practice, the traceability of measurements of a certain physical quantity can be achieved by bringing to a standard that reproduces the unit of this quantity. Usually, it is mutually recognized standards of the leading national metrological institute.

There is another approach, according to which traceability is achieved by the implementation of intrinsic [12] standards. The approach proposed by scientists from Germany can be considered close to it. It consists [13] in the complex study f.i. in TU-Ilmenau of fairly broad issues with help of a specially designed computer that provides a highly stable frequency supply of electronic chips (permissible error of frequency reproduction does not exceed $10^{-16}$ ). Since measurements of standards are performed as frequency dependencies, this contributes to the on-site achievement of such high accuracy of experimental technical equipment, which eliminates the main problem of metrological verification usually including the delivery of verified units to national/regional certification centers and back.

This approach to the problem of ensuring traceability of measurements meets today's requirements and is one of the most important aspects of metrological support, which is the establishment and application of metrological norms, as well as the development and manufacture of metrological instruments necessary to achieve the needed traceability and accuracy. Another, no less important, aspect of metrological support is to ensure the required accuracy of measurements, which is relevant for CPSs. Indeed, many technological processes can be implemented only with the most accurate control of parameters at every stage of production (e.g. microelectronics production).

CPS, consisting of scattered hardware components and software, is designed to obtain information about the course of physical processes in controlled objects, including data storage, transmission, processing, and generation. They are implemented in the lines of production, energetics, etc., become an integral part of health-care, treatment, and rehabilitation systems. These objects monitor the numerical parameters of timevarying processes. Here, the measurement information must be obtained with the specified metrological parameters. Spatial scattering of measuring channels, possible changes in operating conditions, and degradation of measuring circuit parameters lead to a significant deterioration of the metrological properties of the considered 
channels. Therefore, monitoring them becomes essential.

Organizationally, it is important to support and even supervise the CPS's operation by leading metrological laboratories. For this purpose, at the high levels of complex and multi-parameter CPS, an IT metrological service (metrological supervisor) is offered.

Receiving and processing of measuring information in CPSs is carried out by multichannel measuring means [14-15] containing smart sensors, connecting lines or without them in wireless sensor networks, channel switches, and secondary measuring means (Fig.2). The low level of output signals leads to the need to use in the multichannel measuring means' input scaling units or to the normalization of the sensor signals [16]. The normalized or scaled signal is converted into digital code $N_{X}$ and fed to the controller of the considered means. Verification of information-measuring subsystem in the laboratory becomes senseless due to the requirement of its dismantling. In the case of separate verification of the measuring transducer and channel, the operation of modems, communication lines, and the central computer should be checked in-situ.

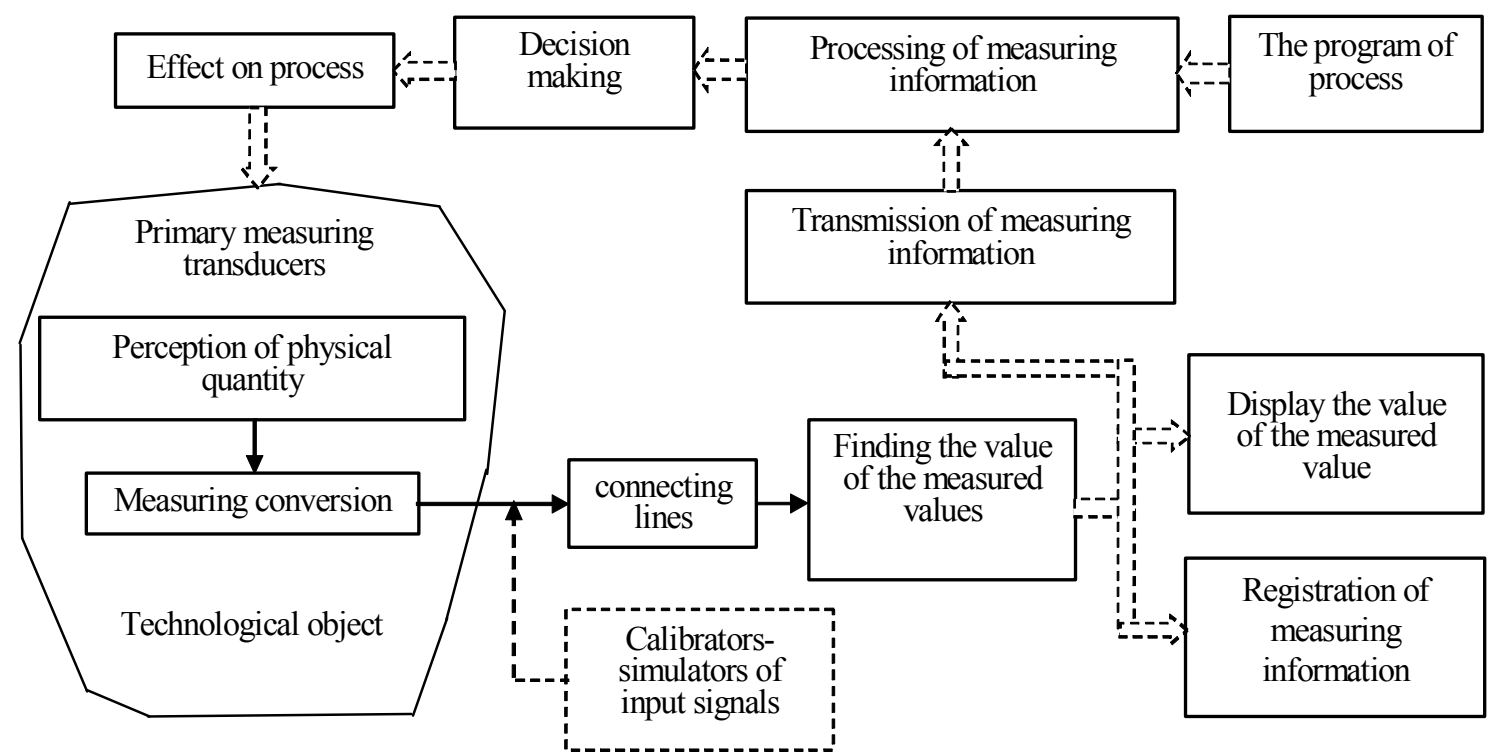

Fig.2. Scheme of multichannel information-measuring subsystem

\subsection{Metrological support of measuring channels}

Means of measuring physical quantities can be implemented as separate devices and/or built into a specific technological product, as separate measuring channels. Their number tends to increase; due to dispersion of location, the optimization of metrological support becomes urgent. Since the considered subsystems are mostly virtual, the revision of traditional verification methods is aimed at their on-site implementation. To perform it, the codecontrolled measures of physical quantities with appropriate software are being developed [17]. Moreover, the verification of complex MCs have required specific codecontrolled measures, with the possibility of their placement directly in the technological objects. An existing network is used as a highway to send measurement information without the additional cables.

\subsection{Measurement quality management}

Traceability, accuracy, precision, uncertainty, and systematic bias are the major components of a measurement quality management system. The main provisions on metrological support of development, production, testing, and operation of the measuring channels, used with the production or research cycle, or other activity, are regulated by [18]. The regulated basis of metrological support is a system of national and working standards of units of physical quantities, comparators for transmitting the size of the considered units, specimens of composition and properties of substances and materials as well as the working measuring instruments.

Therefore, regulations recommend the introduction of methods of control of measurement processes, based on regular inspection and appropriate analysis of control stages and obtained measurement data. This is applied at every level of measurements, from calibration of standards by an external metrological laboratory to the production of regular inspections. Measurement process monitoring procedures can detect non-ordinary deviations in the measurement process; identify problems with measurement repeatability; quantify adjustment factors to bring the test results to standard conditions for any drift of the measured signals; identify irregular bias, including cyclical ones; provide the necessary documentation to meet quality assurance requirements. 
The verification and calibration of measuring instruments are carried out based on special verification schemes, which record the means, methods, and accuracy of transmission of the unit size of the considered value from the national to the working standards and working measuring instruments. For verification of tested measuring instruments, a few methods of their direct comparison regarding the standard measuring instrument of the same type or/and direct measuring of the output signal of multivalued measure by the tested instrument and determining an error as $\Delta_{E}=X_{I}-X_{N}$.

In practice, the measurement process is monitored to achieve technological safety (e.g, the nuclear power plants) or ensure the guaranteed quality of products, goods, and services. The metrological support system must provide guarantees that the measurements (performed with help of the measuring equipment within the inter-verification interval) are sufficiently accurate for the task [19] (Fig.3).

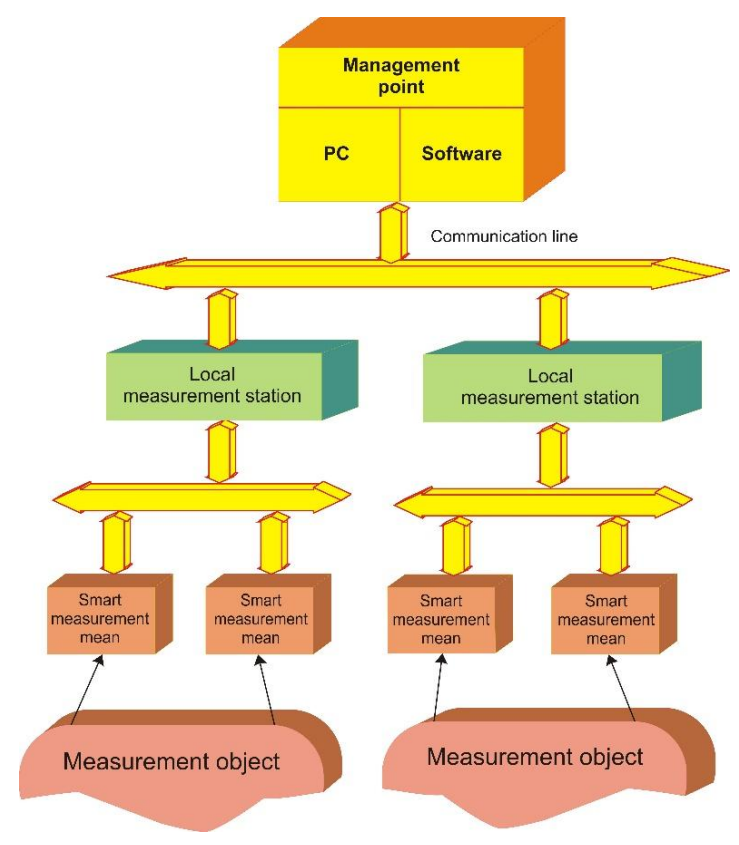

Fig.3. Metrological support system with 2 separate measuring channels

First, in the current issue, it could be considered the qualitative difference in the goals we try to achieve at various stages of the life-cycle of measuring instruments and whole CPS. According to [20] the initial calibration of a sensor on completion of its manufacture is aimed at establishing and specifying the parameters corresponding to a "normal condition" of the sensor. At the stage of online operation, this aim consists in revealing a metrological failure, i.e. deviation of the parameters from their initial values registered at the initial calibration.

Second, the measuring instruments are metrologically tested for the direct consumer of single or small samples compared to the general set of samples. The law of distribution of the measured parameter for the set of samples submitted for periodic inspection differs significantly from the law of distribution of the same parameter at the time of the release of these samples from the production cycle and can hardly be studied. The characteristics of this law depend on such factors as the actual operating conditions of a particular mean and its drift, the qualifications of service personnel, and so on. Recommendations for metrological support of the measuring instruments, needed for actual measurements, the results of which are intended to assess the compliance of products or services with the established standards, are given in [21].

Third, however, the document does not set special requirements for factors that can affect the measurement results, such as measurement methods and techniques, staff competence, etc. Particular emphasis is paid on the maximum user-friendliness of the method with frequent (once a day or more) checking of critical parameters by using implemented standards or "black box" made especially for inspection of the particular parameter. If the "black box" detects non-compliance of the studied samples, the latter must be confirmed in full. However, the implementation of such a method is hampered by the difficulty in determining the critical parameters of the measuring instruments and developing a "black box". Despite its possible high reliability, it cannot replace regular metrological confirmation, since the measuring instruments may fail for a parameter that is not measured by a "black box". In addition, the characteristics of the "black box" maybe not be stable enough.

\section{Conclusions}

1. Within the concept of Open-Science Space, there are several ways to achieve reproducible, repetitive, and traceable research results performed by a group of scattered experimenters. These include educational projects, projects for the operation of known and replicated technical equipment, etc., as well as projects implemented on well-known hardware and software platforms such as LabVIEW. They are to some extent based on smart means of measuring and controlling metrological parameters, but not sufficiently provided and supported metrologically.

2. To develop further this concept and ensure the efficient operation of cyber-physical systems, as technological non-demountable objects with high requirements for metrological characteristics, there are several ways of varying complexity, including the implementation of standards/measures of physical quantities and conversion of several metrological characteristics into frequency-dependent with their measurement concerning the output signals of a specialized frequency high-stable device.

3. A relevant way proposed in this work is the development and implementation of portable code-controlled measures of electrical quantities, inherent 
sufficient time and temperature stability, metrologically confirmed in the laboratory that, if necessary, can be delivered to the site of measuring subsystems of the scattered cyber-physical system for its calibration in working conditions and in real-time.

\section{Gratitude}

The authors express their gratitude to the Staff of the Department of Information and Measuring Technologies of Lviv Polytechnic National University.

\section{Conflict of interest}

The authors state that there are no financial or other potential conflicts regarding this work.

\section{References}

[1] Shaping Europe's digital future. [Online]. Available: https://digital-strategy.ec.europa.eu/en/policies/ cloud-computing

[2] Sang C. Suh, U. John Tanik, John N. Carbone, Abdullah Eroglu, Applied Cyber-Physical Systems, Springer Link, 2014.

[3] Open Science Framework, University of Arizona. [Online]. Available: https://data.library.arizona.edu/datamanagement/ services/ open-science-framework-osf.

[4] J. de la Vera, A. Ruiz, G. Blondelle, Assurance and Certification of Cyber-Physical Systems: The AMASS Open Source Ecosystem, ResearchGate, August 2020, Journal of Systems and Software. doi: 10.1016/j.jss.2020.110812

[5] S. Yatsyshyn, B.Stadnyk, Cyber-Physical Systems and Metrology 4.0. Editors, IFSA Publishing, Barcelona, Spain, 2021.

[6] S. Yatsyshyn, B. Stadnyk, Ya. Yanyshyn, "Information Management and IT Innovation in the Measuring Subsystems of Industry 4.0", in Proc. of the 22nd Int. Conf. on Inf. Techn. for Practice, Oct. 10, 2019, Ostrava, Czech Republic, pp. 105-112, 2019.

[7] Cyton Board. [Online]. Available: https://docs. openbci.com/Cyton/CytonLanding/

[8] P. Ramasamy, Sh. Tharanyaa, J. Palanivelu, A. Sathesan, Certain Applications of LabVIEW in the Field of Electronics and Communication, 2021. doi: 10.5772/intechopen.96301.

[9] A. Hartland, "The quantum Hall effect and resistance standards", Metrologia, vol.29, p.175-190, 1992.
[10] R. Benitez, C. Ramirez, J. Vazquez, Sensors calibration for Metrology 4.0, in Proc.of the II Workshop on Metrology for Industry 4.0 and IoT (MetroInd4.0\&IoT), Naples, Italy, 4-6 June 2019, pp. 296-299.

[11] R.Taymanov, K.Sapozhnikova, "Metrological selfcheck of sensors", ResearchGate, Feb. 2011.

[12] B. Jeckelmann, B. Jeannere, "The Quantum Hall Effect as an Electrical Resistance Standard", Seminaire Poincare, 2, pp.39-51, 2004. http://www.bourbaphy.fr/ jeanneret.pdf.

[13] H. Chen, V. Jungnickel, V. Pohl, C. von Helmolt, "A multicode space-frequency RAKE receiver", IEEE Xplore, Sept. 2004; Asilomar Conference on Signals, Systems and Computers, 2004, At: Pacific Grove, CA, USA, Vol.1, DOI: 10.1109/ACSSC.2004.1399219.

[14] E. Budylina, A. Danilov, N. Ordinartseva, "Method of calibrating measuring channels of measuring systems under operating conditions", , in Proc. of the AMCTM-2017.

[15] M. Jurčević, H. Hegeduš, M. Golub, "Generic System for Remote Testing and Calibration of Measuring Instruments: Security Architecture", Measurement Science Review, vol.10, no.2, p.50-55, 2010.

[16] K. Okorn, M. Hannigan, "Improving Air Pollutant Metal Oxide Sensor Quantification Practices through: An Exploration of Sensor Signal Normalization", Multi-Sensor and Universal Calibration Model Generation, and Physical Factors Such as Co-Location Duration and Sensor Age, Atmosphere, no.12, p.645, 2021. https://doi.org /10.3390/atmos 12050645 https://www.mdpi.com/journal/atmosphere

[17] R. Müller, "Calibration and Verification of Remote Sensing Instruments and Observations", Remote Sensing, iss.6, pp. 5692-5695, 2014.

[18] ISO 10012:2003(en). Measurement management systems - Requirements for measurement processes and measuring equipment, 2003.

[19] R. Sargent, "Validation and verification of simulation models", IEEE Xplore, in Proc. of 2004 Winter Simulation Conf., 2004. https://ieeexplore.ieee.org/xpl/ conhome/9441/proceeding

[20] K. Sapozhnikova, R. Taymanov, "Sensor Devices with High Metrological Reliability", ResearchGate, May 23, 2014. https://www.researchgate.net/publication/221917376.

[21] A. Danilov. New trends in calibration of the measuring systems, in Proc. 7th Int. Conf. "Metrology, Inform.-meas. Techn. \& Systems", 2020. http://umj.metrology.kharkov.ua/issue/view/11766 